\title{
OVERLAYING SURFACE MESHES, PART I: ALGORITHMS
}

\author{
XIANGMIN JIAO* and MICHAEL T. HEATH ${ }^{\dagger}$ \\ Computational Science and Engineering, University of Illinois at Urbana-Champaign \\ Urbana, IL 61801, USA \\ *iiao@uiuc.edu \\ ${ }^{\dagger}$ heath@uiuc.edu
}

Received 6 Feburary 2003

Revised 19 Feburary 2004

Communicated by B. Chazelle

\begin{abstract}
We describe an efficient and robust algorithm for computing a common refinement of two meshes modeling the same surface of arbitrary shape by overlaying them on top of each other. A common refinement is an important data structure for transferring data between meshes that have different combinatorial structures. Our algorithm is optimal in time and space, with linear complexity, and is robust even with inexact computations, through the techniques of error analysis, detection of topological inconsistencies, and automatic resolution of such inconsistencies. We present the verification and some further enhancement of robustness in Part II.
\end{abstract}

Keywords: Computational geometry; mesh overlay; common refinement; topological consistency; edge intersection; robustness; inexact computation; data transfer.

\section{Introduction}

In many scientific applications, one must often transfer data between differing mesh representations of a surface model. Such meshes are in general nonmatching, meaning that their combinatorial structures differ and their geometric realizations may have gaps between them or interpenetrate each other due to discretization or rounding errors. Examples of such applications include fluid-solid interaction, adaptive meshing, multigrid methods, texture mapping, and shape morphing. In physically meaningful applications, such as fluid-solid interaction, data transfer must be numerically accurate and physically conservative. In this paper, we introduce a data structure called a common refinement of two meshes, which is crucial for such datatransfer tasks. Using this data structure, we have developed a data-transfer method that is both accurate and conservative, which we report elsewhere. ${ }^{1}$

A common refinement of two meshes is a mesh composed of polygons that subdivide the polygons of both input meshes simultaneously. Each cell of a common refinement has two geometric realizations, which in general are different but must be close to each other for physically meaningful data transfer. A common refinement 


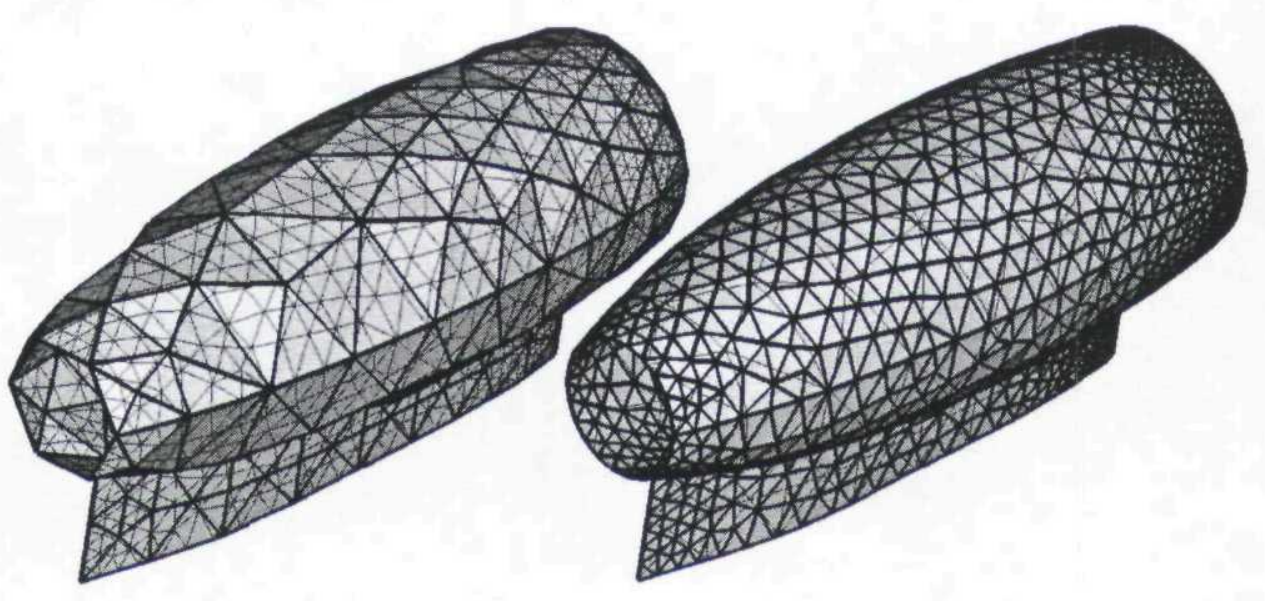

Fig. 1. Common refinement of two surface meshes modeling engine nacelle of Falcon aircraft.

defines, and allows efficient query of, a unique nearby corresponding point on one surface for every point on the other. Figure 1 shows an example of a common refinement of two triangular meshes modeling an engine nacelle of an aircraft. The thick lines are the edges of the input meshes. The refined meshes formed collectively by the thick and thin lines in either image are isomorphic to each other (i.e., they have the same combinatorial structure), and are the realizations of the common refinement on the two input meshes, respectively.

A common refinement of surface meshes has been deemed desirable but too complex or impractical to compute, and has generally been avoided by application scientists and engineers. ${ }^{2,3,4}$ It is indeed challenging to compute because in general the geometric realizations of the meshes define distinct surfaces. It is even more challenging to develop a robust algorithm because the primitives involved are necessarily nonlinear and can be solved only approximately, and one must detect and resolve the potential inconsistencies caused by these inexact computations. A surface model with large curvatures, ridges, corners, or boundaries, such as the one in Figure 1, adds further complexity.

This paper presents an efficient and robust algorithm for constructing a common refinement of two meshes modeling a 2-manifold with boundary by overlaying them on top of each other. In this method, we first construct a nearly orthogonal projection between two mesh surfaces that gives a continuous and one-to-one correspondence between the points of their respective geometric realizations. Based on this projection, intersections of edges are defined, which are then used to compute the common refinement. The primitives of our algorithm involve nonlinear equations that can be solved only approximately by iteration. The inevitable presence of numerical errors can introduce potential numerical and topological inconsistencies. Our algorithm achieves robustness through a combination of techniques, including 
error analysis and associated tolerancing, detection of inconsistencies, and automatic resolution of such inconsistencies using topological operations. We present the verification and some further enhancement of robustness in a companion paper (Part II). Our algorithm works for meshes containing triangular or quadrilateral facets, and hence is applicable to most surface meshes used in practice. Furthermore, it is optimal in time and space, with linear complexities.

Although a few algorithms have been previously proposed for overlaying meshes, they address the problem in much more limited and idealized settings. For example, a number of algorithms have been described for overlaying two triangular meshes on a plane ${ }^{5}$ or on a sphere,,$^{6,7}$ where edge intersections were computed analytically and exact arithmetic and symbolic perturbation were assumed for simplicity. In addition, these previously published algorithms have suboptimal worst-case complexities, with $O(k+n \log n){ }^{5,6}$ or $O(n+k \log k),{ }^{7}$ where $n$ is input size and $k$ is output size. A problem related to mesh overlay is map overlay, which overlays two subdivisions of a plane,$^{8}$ for which there are some well-known results. ${ }^{9,10,11,12}$ Both problems share edge intersection as a primitive and share similar data structures, but map overlay handles objects embedded only in $\mathbb{R}^{2}$.

An important achievement of our algorithm is its robustness even with inexact computations. Robustness issues have drawn considerable attention in computational geometry. ${ }^{13,14,15,16,17,18}$ Some previous work has focused on techniques to cope with degeneracies, ${ }^{13,16,18}$ but a fundamental limitation of such approaches is their dependence on exact computations. Some other work has been conducted on robustness with floating-point arithmetic through interval arithmetic, ${ }^{15}$ but near degeneracies are still ambiguous for such techniques and arbitrarily large uncertainty intervals can occur for edge intersections. Our approach is closer to the data normalization method, ${ }^{17}$ in that we assume some well-shapedness conditions about the input. The more recent work on robust arrangements through snap rounding ${ }^{19,20}$ also share some similarities with our techniques in achieving robustness.

The remainder of the paper is organized as follows. Section 2 introduces some background concepts, notation, and data structures. Section 3 introduces the basic primitives of the algorithm, namely point projection and edge intersection. Section 4 describes the basic algorithm using idealized exact arithmetic. Section 5 addresses robustness of the primitives when using inexact computations. Section 6 describes the resolution of inconsistencies for smooth surfaces. Section 7 contains a concluding discussion.

\section{Definitions}

\subsection{Surface meshes}

We start by defining the topological and geometric objects involved in our problem. Most of the terms can be found in textbooks on differential or computational geometry. ${ }^{21,22}$ The basic objects of interests are cells (or faces) of dimension 0,1 , or 2 , which are points, closed topological intervals, and closed topologi- 
cal disks, respectively. We call the 0 -dimensional cells vertices, the 1 -dimensional cells edges, and the 2-dimensional cells facets. Each cell $\sigma$ has a geometric realization (or simply realization) in $\mathbb{R}^{3}$, denoted by $|\sigma|$, which is the point set $\left\{\sum_{i} N_{i} x_{i} \mid \sum_{i} N_{i}=1\right.$ and $\left.N_{i} \geq 0\right\}$, where the $x_{i}$ are the vertices of the cell, and the $N_{i}(\xi, \eta): \mathbb{R}^{2} \rightarrow \mathbb{R}$ are the associated shape functions (such as those used in finite element analysis) or barycentric coordinates, which are functions of the local coordinates $(\xi$ and $\eta$ ) of the cell. The realization of an edge is the line segment between its two vertices, and that of a triangle or a quadrilateral is a patch of a plane or a bilinear surface bounded by their edges. An interface mesh, or surface mesh, is a collection of these cells and is required to be a pure complex, which means that the boundary of each facet is a finite union of edges, each edge or vertex belongs to at least one facet, and any two facets either are disjoint, intersect in a single vertex, or intersect along a single edge. The realization of a mesh $M$, denoted by $|M|$, is the union of the realizations of the cells in $M$, which is also called the underlying space of $M$. For convenience, we sometimes refer to $|M|$ as a mesh surface.

To unify the treatments of various cases that will arise our discussion, we also need the following additional topological definitions and notation, most of which can be found in the textbook by Edelsbrunner. ${ }^{22}$ If a cell $\sigma$ is contained in another cell $\tau$, we say $\sigma \leq \tau$. The closure of a subset $S$ of a mesh $M$ is the set of cells that are contained in the cells in $S$, i.e., $\bar{S}=\{\sigma \in M \mid \sigma \leq \tau \in S\}$. The star of a cell $\sigma$, denoted by St $\sigma$, is the set of cells that contain $\sigma$, i.e., St $\sigma=\{\tau \in M \mid \sigma \leq \tau\}$, and the star of a subset $S$ is defined as $\bigcup_{\sigma \in S} \operatorname{St} \sigma$ and denoted by St $S$. The link of a cell $\sigma$, denoted by $\mathrm{Lk} \sigma$, is the set of cells in $\overline{\mathrm{St} \sigma}$ but not in St $\bar{\sigma}$, i.e., Lk $\sigma=\overline{\mathrm{St}} \sigma-\mathrm{St} \bar{\sigma}$. A related concept is the completion of the star of $\sigma$, which we define as $\overline{\mathrm{St}} \sigma-\mathrm{St} \sigma$ and denote by $\operatorname{Cs} \sigma$. If $\sigma$ is a vertex, its link and the completion of the star are the same. If $\sigma$ is a border edge or vertex, $\operatorname{Cs} \sigma$ is a linear sequence of edges and vertices; otherwise, $\operatorname{Cs} \sigma$ is a cyclic sequence of edges and vertices.

\subsection{Common refinement}

A mapping $f: X \rightarrow Y$ is continuous if the preimage of every open set in $Y$ is an open set in $X$. Two sets $X$ and $Y$ are homeomorphic, denoted by $X \approx Y$, if there is a bijective and continuous mapping $f: X \rightarrow Y$ whose inverse is also continuous; such an $f$ is called a homeomorphism. A mesh $R$ is a refinement (or subdivision) of a mesh $M$ if their geometric realizations $|R|$ and $|M|$ are homeomorphic and every cell of $M$ is partitioned into one or more cells of $R$. Given two homeomorphic meshes, a common refinement of them is a mesh that is a refinement of both given meshes.

Let one of the given meshes, denoted by $B$, be colored blue, and the other, denoted by $G$, be green, and their cells be referred to as blue or green cells, respectively. We require each facet of $B$ and $G$ to be either a triangle or a quadrilateral. The facets of their common refinement $R$, however, can have larger numbers of edges. In our applications, the meshes are portions of surfaces of three-dimensional 
domains. We therefore assume that an input mesh is a 2-manifold with boundary, which means every point in its realization has a neighborhood homeomorphic to a plane or a half-plane. Nonmanifold surfaces arising from CAD modeling are typically composed of manifold subsurfaces, and the concept and the algorithm that we will describe can be extended to such nonmanifold surfaces by applying it to the subsurfaces. Without loss of generality, we also assume each input mesh is connected. Sometimes, we assume a mesh is well-shaped, so that no facet has very small angles, and the mesh approximates the underlying surface reasonably well.

We refer to the cells of the common refinement as subcells, and in particular, refer to the 0-, 1-, and 2-dimensional subcells as subvertices, subedges, and subfacets, respectively. Each subcell $r \in R$ is contained in some cells in $B$ or in $G$, which are the blue or green host cells of $r$. We define the blue or green parent of a cell $r \in R$ (and in general that of a set of cells in $R$ ), denoted by $\mathrm{B} r$ or $\mathrm{G} r$, to be the lowest dimensional blue or green cell, respectively, that contains $r$. Note that every subcell of a common refinement has two geometric realizations, one contained in the realization of either of its parents. By construction, the interior of a subcell is contained in the interiors of its parents. We say that a common refinement $R$ is minimal if no two subcells in $R$ share the same blue and the same green parent. Such a common refinement is minimal in the sense that we cannot reduce the output size by merging the subcells without changing their parents.

\subsection{Conforming homeomorphism}

Let $f:|B| \rightarrow|G|$ be a homeomorphism. We define the intersection of $b \in B$ and $g \in G$ induced by $f$, denoted by $b \cap_{f} g$, to be the topological point set that corresponds to $f(b) \cap g$, or equivalently $b \cap f^{-1}(g)$. In other words, $b \cap_{f} g$ has two geometric realizations, one on $|G|$ and the other on $|B|$, between which $f$ is a homeomorphism. We say that a homeomorphism $f$ is conforming if for every pair of cells $\langle b \in B, g \in G\rangle$, the intersection $b \cap_{f} g$ is either the empty set, a point, an interval, or a topological disk. For a conforming homeomorphism, the intersection of a blue edge and the boundary of a green facet contains at most two discrete points or contains one interval, and vice versa. Note that the identity mapping is always conforming for two flat meshes that consist of convex polygons and have the same underlying space. Figure 2 shows an example in which the identity mapping is not conforming between the two meshes $B$ and $G$ composed of the solid and dashed edges, respectively, because the solid edge in the middle intersects the shaded green facet along two intervals.

Lemma 1. Pairwise Intersections. Given two meshes $B$ and $G$ and a conforming homeomorphism $f:|B| \rightarrow|G|$, the pairwise intersections of the blue and green cells induced by $f$ form a minimal common refinement of $B$ and $G$.

The proof is presented in Appendix A. We refer to the common refinement formed by the pairwise intersections induced by a conforming homeomorphism as 


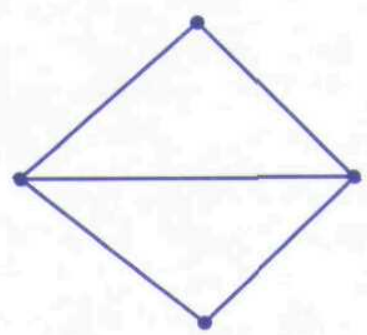

$B$

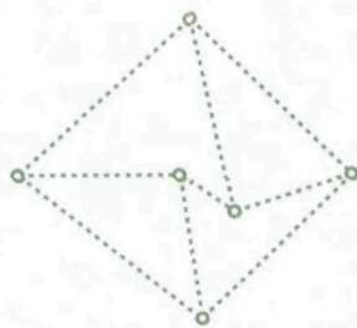

G

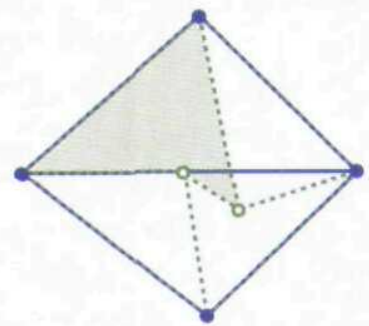

overlay

Fig. 2. Example where identity mapping is not conforming homeomorphism.

the overlay of $B$ and $G$, and refer to the process of constructing this common refinement as overlaying the meshes. This definition is consistent with the traditional notion of overlaying two subdivisions of a plane, ${ }^{8}$ except that traditionally the identity mapping serves implicitly as the underlying homeomorphism, whereas in our setting we must define the homeomorphism explicitly and require it to be conforming. We comment that a nonconforming homeomorphism can also induce a minimal common refinement, such as that in Figure 2, and there are infinitely many conforming homeomorphisms that can lead to different overlays. In later sections, we will construct a conforming homeomorphism that is convenient for computing pairwise intersections.

\section{Basic Primitives}

In this section, we define two primitives, namely point projection and edge intersection, which we will need for our mesh-overlay algorithm.

\subsection{Point projection}

Two types of projections are commonly used to correlate points on two surfaces, namely orthogonal projection and closest-point projection. These projections map a point on one surface onto a nearby point on another surface, and vice versa, which is reasonable for some applications. Unfortunately, neither of these projections can possibly define a homeomorphism between two mesh surfaces, because a triangular or quadrilateral mesh surface is in general not smooth at edges or vertices.

We construct a nearly orthogonal projection by interpolation using the shape functions of the green mesh. We first define the projection from $|G|$ to $|B|$, and will use its inverse to define the projection from $|B|$ to $|G|$. For any point $p \in|G|$, we can write its projection $\boldsymbol{q} \in|B|$ as $\boldsymbol{p}+\gamma(\boldsymbol{p}) \boldsymbol{d}(\boldsymbol{p})$, where $\boldsymbol{d}:|G| \rightarrow \mathbb{S}^{2}$ denotes the normalization of $\boldsymbol{q}-\boldsymbol{p}$, and $\gamma:|G| \rightarrow \mathbb{R}$ denotes the length of $\boldsymbol{q}-\boldsymbol{p}$. We construct such a function $\boldsymbol{d}$ by first fixing its values at the green vertices, which can be given by the user or evaluated numerically as the average of the facet normals. 


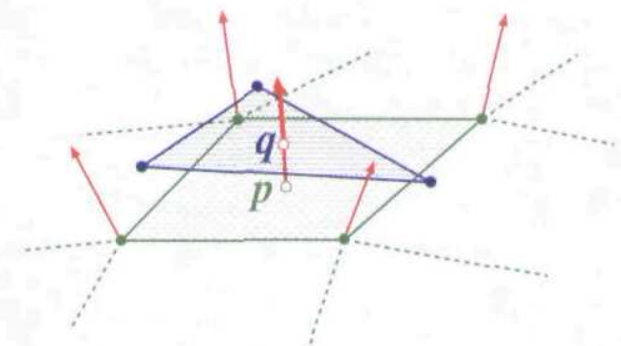

Fig. 3. Point projection from green (lower) to blue (upper) mesh.

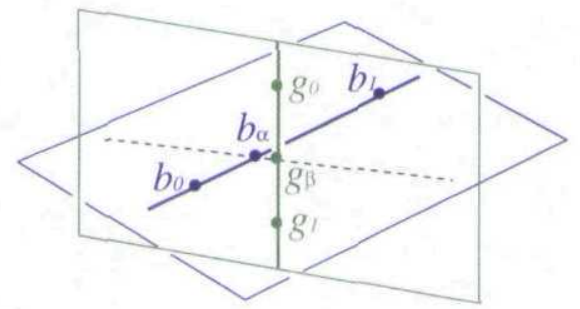

Fig. 4. Illustration of edge intersection.

Let $\boldsymbol{x}_{i}$ denote the $i$ th vertex, and $\boldsymbol{d}_{i}$ denote its associated unit vector. For a point $\boldsymbol{p}=\sum_{i} N_{i} x_{i} \in|G|$, its direction $\boldsymbol{d}$ is then the normalized vector of $\sum_{i} N_{i} d_{i}$, as illustrated in Figure 3. The continuity of $\boldsymbol{d}$ follows from the continuity of the $N_{i}$. This projection has also been used in some similar applications. ${ }^{23}$

The main virtues of this projection are that it is close to orthogonal for reasonably smooth surfaces, its projection directions form a continuous vector field over the surface, and it is convenient to compute. Approximate orthogonality is desirable for accurate data transfer, the main application of common refinement. It is also important for good conditioning of the equations that must be solved for the primitives. The continuity of $\boldsymbol{d}$ makes it possible for the point correspondence to be a homeomorphism. We note that for closed surfaces if the projection is nearly orthogonal, the continuity of $\boldsymbol{d}$ implies that there exists an $\varepsilon$ that depends on the smoothness of $\boldsymbol{d}$, such that the point correspondence is a homeomorphism if the Hausdorff distance ${ }^{24}$ (i.e., the max-min distances between two point sets) between the surfaces is smaller than $\varepsilon$. For now, we shall assume the two meshes are modeling a closed smooth surface, and they are close to each other so that the projection is a homeomorphism. We further assume that the homeomorphism is conforming. These assumptions become invalid for surfaces with ridges, corners, or nonmatching boundaries, which we will address in Part II. Note that when the projection is a homeomorphism, its inverse exists, which gives the projection from $|B|$ to $|G|$.

This primitive is defined piecewise on the facets of the green mesh, and it is very convenient to evaluate. In particular, the projection of a point $p \in|G|$ onto a facet $b \in B$ is the intersection of $b$ with the line passing through $\boldsymbol{p}$ with direction $\boldsymbol{d}(\boldsymbol{p})$. If $\sum N_{i} x_{i} \in b$ is the projection of $\boldsymbol{p}$, then we can pose the problem as a system of equations

$$
\sum N_{i} x_{i}-p-\gamma d=0
$$

Because $N$ is a function of the two local coordinates of $b$, this system has three equations (one for each component of the physical coordinates) and three unknowns ( $\gamma$ and the local coordinates). The system is linear if the blue facet $b$ is a triangle but is bilinear if $b$ is a quadrilateral. In the latter case, we solve the system using 
Newton's method, which converges quadratically when $\boldsymbol{d}$ is nearly orthogonal to the facet. The primitive returns the local coordinates of the projection and reports whether $\boldsymbol{p}$ projects to the interior, an edge, a vertex, or the exterior of the facet, identified from the barycentric coordinates.

The projection from a point $q \in|B|$ to a green facet $g \in G$ can be computed similarly. Let the point $\sum N_{i} x_{i}$ be its projection in $g$ whose projection direction is $\sum N_{i} d_{i}$. We then have the system of nonlinear equations

$$
\sum N_{i} x_{i}-q-\gamma \sum N_{i} d_{i}=0
$$

which also has three unknowns and can be solved similarly.

\subsection{Edge intersection}

Another primitive for mesh overlay is to compute the intersection of a blue edge $b \in B$ with a green edge $g \in G$. Again, the intersection has two realizations, one in $|B|$ and one in $|G|$. This primitive determines both realizations and reports whether the intersection is in the interior, at a vertex, or the empty set.

Let the blue edge be $b=\boldsymbol{b}_{0} \boldsymbol{b}_{1}$ and the green edge be $g=\boldsymbol{g}_{0} \boldsymbol{g}_{1}$, and let the projection directions at $g_{0}$ and $g_{1}$ be $\boldsymbol{d}_{0}$ and $\boldsymbol{d}_{1}$, respectively. We parameterize $b$ by $\boldsymbol{b}_{0}+\alpha\left(\boldsymbol{b}_{1}-\boldsymbol{b}_{0}\right)$, and parameterize $g$ by $\boldsymbol{g}_{0}+\beta\left(\boldsymbol{g}_{1}-\boldsymbol{g}_{0}\right)$. The projection direction of a point in $g$ is then $\boldsymbol{d}_{0}+\beta\left(\boldsymbol{d}_{1}-\boldsymbol{d}_{0}\right)$, and hence

$$
\boldsymbol{b}_{0}+\alpha\left(\boldsymbol{b}_{1}-\boldsymbol{b}_{0}\right)=\boldsymbol{g}_{0}+\beta\left(\boldsymbol{g}_{1}-\boldsymbol{g}_{0}\right)+\gamma\left(\boldsymbol{d}_{0}+\beta\left(\boldsymbol{d}_{1}-\boldsymbol{d}_{0}\right)\right),
$$

which again has three equations and three unknowns. This formulation, unfortunately, is very ill-conditioned when $b$ and $g$ are nearly parallel, which happens frequently in practice, and Newton's method can converge very slowly or fail to converge with this formulation. We hence reformulate the equation as follows. Note that the realization of the intersection point in $g$ is in the plane passing through $b$ with normal direction $\boldsymbol{n}=\left(\boldsymbol{b}_{1}-\boldsymbol{b}_{0}\right) \times\left(\boldsymbol{d}_{0}+\beta\left(\boldsymbol{d}_{1}-\boldsymbol{d}_{0}\right)\right)$, as illustrated in Figure 4 . Therefore, we have the equation

$$
\boldsymbol{n} \cdot\left(\boldsymbol{g}_{0}+\beta\left(\boldsymbol{g}_{1}-\boldsymbol{g}_{0}\right)-\boldsymbol{b}_{0}\right)=0 .
$$

Substituting $\boldsymbol{n}$ and reordering the equation leads to a quadratic equation $c_{2} \beta^{2}+$ $c_{1} \beta+c_{0}=0$, where

$$
\begin{aligned}
& c_{2}=\left(\left(b_{1}-b_{0}\right) \times\left(d_{1}-d_{0}\right)\right) \cdot\left(g_{1}-g_{0}\right), \\
& c_{1}=\left(\left(b_{1}-b_{0}\right) \times d_{0}\right) \cdot\left(g_{1}-g_{0}\right)+\left(\left(b_{1}-b_{0}\right) \times\left(d_{1}-d_{0}\right)\right) \cdot\left(g_{0}-b_{0}\right), \\
& c_{0}=\left(\left(b_{1}-b_{0}\right) \times d_{0}\right) \cdot\left(g_{0}-b_{0}\right),
\end{aligned}
$$

which can be solved analytically. The realization in $b$ is the intersection of the edge $b$ with the plane passing through $g$ having normal $\boldsymbol{l}=\left(\boldsymbol{g}_{1}-\boldsymbol{g}_{0}\right) \times\left(\boldsymbol{d}_{0}+\beta\left(\boldsymbol{d}_{1}-\boldsymbol{d}_{0}\right)\right)$. Therefore, the parameter $\alpha$ is the solution to the linear equation

$$
\boldsymbol{l} \cdot\left(\boldsymbol{b}_{0}+\alpha\left(\boldsymbol{b}_{1}-\boldsymbol{b}_{0}\right)-\boldsymbol{g}_{0}\right)=0
$$


which can be solved after we have obtained $\beta$, provided $\boldsymbol{l} \cdot\left(\boldsymbol{b}_{1}-\boldsymbol{b}_{0}\right) \neq 0$.

The intersection is in the interior of $b$ if $\alpha \in(0,1)$, at a vertex if $\alpha=0$ or 1 , in the exterior otherwise; similarly for $\beta$. A solution corresponds to an actual edge intersection if $\alpha \in[0,1]$ and $\beta \in[0,1]$ simultaneously. Note that the above equations can have zero, one, two, or infinitely many solutions. We will take special care in our algorithm to avoid having infinitely many solutions. When two edges are nearly parallel or $\boldsymbol{d}_{0}$ and $\boldsymbol{d}_{1}$ differ significantly, the equation can be very ill-conditioned and leads to zero, one, or two solutions. For now we assume the solver is reliable and returns a single valid solution, and defer robustness issues to Section 5 .

\subsection{Data structure}

We represent a mesh using the doubly-connected edge list (DCEL) data structure, ${ }^{8}$ also known as the half-edge structure. ${ }^{25}$ This data structure represents each edge by two directed half edges, each of which is said to be the twin of the other. A half edge $h$ has an origin vertex, denoted by org $h$, and a destination vertex, denoted dst $h$. The origin of a half edge is the destination of its twin, and vice versa. We say that a half edge and its origin are incident. Each half edge bounds the facet to its left, and a half edge and the facet it bounds are said to be incident. The incident half edges of a facet form a counterclockwise ring, and the incident half edges of a vertex and their twins form a clockwise ring. For each edge $e$, we choose one half edge as its primary half edge, and the origin/destination of $e$ is the origin/destination of the primary half edge. The DCEL representation allows one to walk through the incident half edges of a vertex or a facet efficiently. Some other data structures, such as the quad-edge structure ${ }^{26}$ and the winged-edge structure, ${ }^{27}$ also have similar functionality and hence are feasible alternatives.

\section{Overlay Algorithm}

We now describe our algorithm for mesh overlay in a simplified setting that assumes that the surface is closed and smooth and the primitives are evaluated exactly. With these assumptions, we will focus on symbolic arguments and combinatorial properties of the algorithm and ignore the more practical issues that are addressed in Section 5. The algorithm has two phases. In the first phase, we identify all the subvertices of the overlay and their blue and green parents and sort them in their host edges. In the second phase, we use the parent information to determine subfacets of the overlay. In anticipation of generalizing to cases with inexact computations, we take into account possible degeneracies of vertex-vertex, vertex-edge, and edge-edge coincidences in our algorithm.

\subsection{Phase one - locating subvertices}

A subvertex is either a blue or green vertex, or an intersection of a blue and a green edge. We locate the subvertices and their parents in the following three steps: 
(1) locate subvertices along blue edges;

(2) sort subvertices in their green host edges;

(3) determine blue parents for remaining green vertices.

\subsubsection{Step one - locating subvertices along blue edges}

This step locates the subvertices in the blue edges and blue vertices and determines their green parents. To accomplish this, we traverse $B$ in a breadth-first order and for each edge $b \in B$ locate the subvertices in $b$ from one end to the other. Suppose $b$ is a blue half edge and $r_{0}$ is the subvertex corresponding to org $b$, whose green parent is known. We find the other subvertices $r_{1}, \ldots, r_{m}, r_{m+1}=$ dst $b$ in edge $b$ and determine their parents, where $r_{1}, \ldots, r_{m}$ are in the interior of $b$ sorted from $\operatorname{org} b$ to dst $b$. We locate $r_{i}$ in increasing order of $i$ and intersect $b$ with the green cells that can possibly host $r_{i}$. This operation is repeated until all blue edges have been processed. To start the algorithm, it is necessary to know the green parent of some blue vertex, which can be obtained by brute force.

We now determine which green cells are topologically consistent with the green parents of the other subvertices and hence are candidates for the green parent of $r_{i}$. This identification is crucial for the efficiency of the algorithm, and also for developing robust algorithms with inexact computations in the next section. Let $g_{i}$ denote the green parent of a subvertex $r_{i}$. We note the following lemma, whose proof is given in Appendix B.

Lemma 2. Host Candidate. Given a conforming homeomorphism,

$$
g_{i+1} \in \begin{cases}\operatorname{Cs} g_{i} & \text { if } 0=i \neq m, \\ \operatorname{Cs} g_{i}-\overline{g_{i}}-\bigcup_{j<i} \overline{\operatorname{St} g_{j}} \text { if } 0<i<m, \\ \overline{\operatorname{St} g_{i}}-\overline{g_{i}}-\bigcup_{j<i} \overline{\operatorname{St} g_{j}} \text { if } i=m .\end{cases}
$$

The Host Candidate Lemma characterizes the topological relationship between the green parents of the subvertices in a blue edge $b$. Note that the lemma specifies only the relationship between $g_{i}$ and $g_{j}$ for $i>j$, which is sufficient to characterize fully the relationship between all the subvertices in $b$, because the relationship for $i<j$ is given implicitly, as stated in the following lemma.

Lemma 3. Implicit Candidate. If the condition for the Host Candidate Lemma holds for all $0 \leq i \leq m$, then

$$
g_{i} \in \begin{cases}\operatorname{Cs} g_{i+1} & \text { if } i=m, \\ \operatorname{Cs} g_{i+1}-\overline{g_{i+1}}-\bigcup_{j>i+1} \overline{\operatorname{St} g_{j}} & \text { if } 0<i<m, \\ \overline{\operatorname{St} g_{i+1}}-\overline{g_{i+1}}-\bigcup_{j>i+1} \overline{\operatorname{St} g_{j}} & \text { if } 0=i \neq m .\end{cases}
$$

This lemma is proved in Appendix C. A corollary of the Host Candidate Lemma is that if we define $g_{-1}$ to be $\emptyset$, then $b$ intersects Cs $g_{i}-\overline{\text { St } g_{i-1}}$ at most once for $i \geq 0$. This is because $b$ intersects Cs $g_{i}$ at most twice, one before $r_{i}$ and one after $r_{i}$. The one before $r_{i}$, if it exists, must be contained in $\overline{\operatorname{St} g_{i-1}}$; the one after $r_{i}$, 

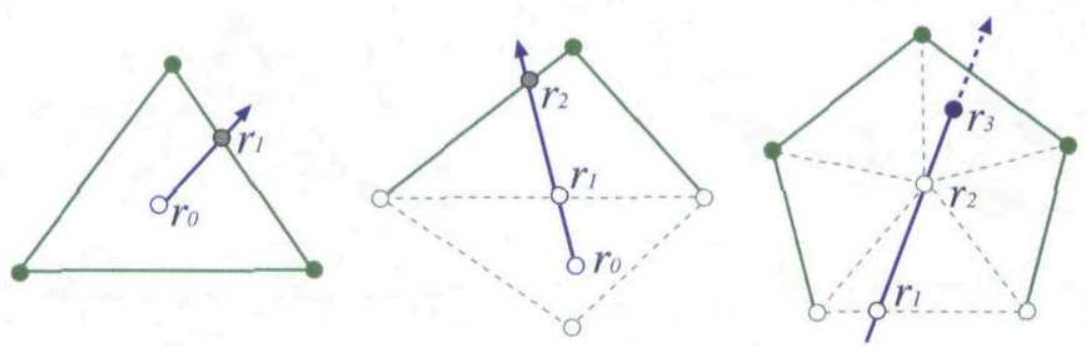

Fig. 5. Intersecting blue (darker) edge with green (lighter) edges in Cs $g_{i}-\overline{\mathrm{St}} g_{i-1}$ to locate subvertex $r_{i+1}$. From left to right, $i$ is 0,1 , and 2 respectively. Solid edges and vertices are candidates for host of next intersection.

according to the lemma, must not be in $\overline{\mathrm{St} g_{i-1}}$. This observation leads us to an efficient algorithm. Specifically, we compute the intersection of $b$ with the green edges in $\mathrm{Cs} g_{i}-\overline{\mathrm{St} g_{i-1}}$. If there is an intersection, it must be $r_{i+1}$. Otherwise, dst $b$ must be $r_{i+1}$, which is contained in St $g_{i}$. Figure 5 illustrates these situations. We note particularly the case when $b$ is concurrent with a green edge, as illustrated by the example in Figure 6, where $b$ overlaps with the green edge bounded by $r_{i} r_{i+1}$. Because $r_{i} \in b$, but $r_{i}=g_{i} \notin \mathrm{Cs} g_{i}$, the green edge that is concurrent with $b$ cannot be in $\mathrm{Cs} g_{i}$. This effectively avoids computing the intersection of two overlapping edges, which otherwise would lead to infinitely many solutions for the edge intersection primitive.

\subsubsection{Step two - sorting subvertices in green edges}

After obtaining all the blue and green edge intersections, we must sort them in their green parent edges. It is feasible to use a comparison-based sorting algorithm (such as quicksort), but we are reluctant to do so because the correctness of the algorithm would then crucially depend on the reliability of the numerical solutions of edge intersections. Instead, we use the parent information and mesh topology to sort the intersections. This allows easy generalization to inexact computations and also enables better efficiency.

Observe that two adjacent subvertices in a green edge must be contained in a common blue facet. Therefore, we traverse the blue facets to identify the adjacency of subvertices in green edges. Specifically, for each blue facet, we group the subvertices in its edges and vertices based on their green parents, with a green edge containing at most two subvertices in its group. If a group has two subvertices, we identify the one that is closer to the origin of the green edge as the predecessor of the one that is farther, and link them with each other, as illustrated in Figure 7. After we have processed all blue facets, the subvertices in each green edge are connected together to form a sorted linked list. 


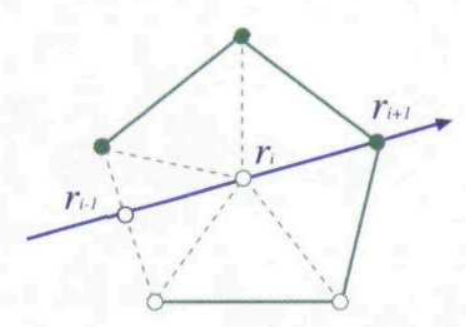

Fig. 6. Green edge $r_{i} r_{i+1}$ is disqualified to host $r_{i+1}$ by Host Candidate Lemma, avoiding computation of intersection of concurrent edges.

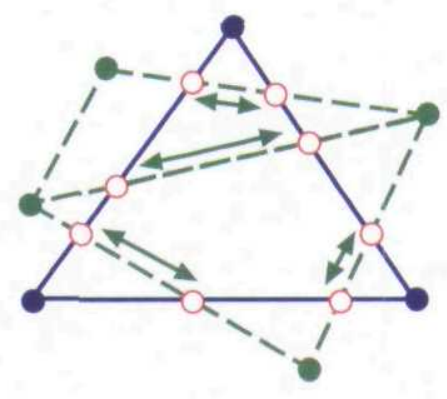

Fig. 7. Connecting each pair of subvertices that are on boundary of same blue (solid) face and also in same green (dashed) edge.

\subsubsection{Step three - projecting green vertices}

We still must determine the blue parents for the green vertices that are not in a blue edge or a blue vertex. This step can be considered the dual of step 1, and there are at least two ways to do it. One way is to repeat step 1 but interchange $B$ and $G$ in traversing the nodal graph. Another way is to traverse the dual graph of $B$ to visit every blue facet, which is the approach we take. For each blue facet $b$, we start from the subvertices in its edges and vertices to traverse the green vertices that can project onto the blue facet based on the Host Candidate Lemma, and then test whether they indeed project onto $b$ using point projection. If so, the blue parent of the green vertex is $b$, and we continue to visit its neighbor green vertices. Otherwise, we ignore the green vertex. This approach requires a global traversal of $B$ and a local traversal of $G$, which is consistent with step 1 , and therefore it has greater potential for generalization, such as to the case where $B$ is a subset of $G$.

\subsubsection{Analysis of complexity}

Let $n$ be the total number of vertices, edges, and facets in $B$ and $G$. Let the degree of a subvertex $v$, denoted by $d(v)$, be the number of blue and green facets that contain $v$, and let $k$ be the sum of degrees of all subvertices, which is typically $O(n)$ as we argued in a previous work. ${ }^{28}$ We now show that this phase of the algorithm takes $O(n+k)$ time and space. The first step traverses the nodal graph of $B$ to locate subvertices in its incident blue edges. During the traversal, when visiting a blue vertex, we visit its incident blue half edges in clockwise order. To locate a new subvertex in a half edge $b$, we rotate also in clockwise order along the green edges and stop the rotation after we find the subvertex. When processing the next incident blue edge, we continue the rotation on green edges starting from where we just stopped. This simultaneous clockwise rotation guarantees that the cost associated with a subvertex $v$ is $O(d(v))$, and the total cost is then the sum of the degrees of subvertices, which is $O(n+k)$. The argument for steps 2 and 3 is similar. 
Note that if step 2 uses a comparison-based sort, the worst case complexity would be $O(n \log n+k)$, which is suboptimal.

\subsection{Phase two - determining subfacets}

Phase 1 of the algorithm has determined all the subvertices and sorted them in the blue and green edges. The subedges of the overlay are the intervals in the blue and green edges between the subvertices. Phase 2 connects these subvertices and subedges to determine subfacets of the overlay. We describe two efficient ways for doing this, neither of which involves numerical computations but uses only the parent information of the subvertices obtained from the previous phase. The first approach constructs a DCEL structure for the overlay in linear time, which has more theoretical interest because of its optimality. The second approach takes a blue or green facet and enumerates the subfacets contained in it, which has more practical interest because of its minimal memory requirement.

\subsubsection{Constructing DCEL structure}

The first approach constructs a DCEL representation for the overlay, which contains all its connectivity information. We proceed in two steps. First, construct a DCEL structure for the refinement graph $\widehat{B}$ of $B$, of which the vertices are the subvertices contained in edges or vertices of $B$, and two vertices are adjacent if they are contained in a common blue edge. We define the blue or green parent facet of a half edge in $\widehat{B}$ as the facet in $B$ or $G$ to its left. Similarly for $G$ and $\widehat{G}$. Second, merge the edge rings of each subvertex in $\widehat{B}$ and $\widehat{G}$ to obtain the DCEL of the overlay. This is equivalent to merging two sorted lists of points on a topological circle: the circle is the link of a subvertex, and the points are its adjacent subvertices in $\widehat{B}$ and $\widehat{G}$, which form two sorted lists. The merging is done by rotating around the two circles synchronously, which takes time proportional to the degree of the subvertex. The parent information of the half edges is used to determine the order. The two graphs are merged by looping over all the subvertices in both $\widehat{B}$ and $\widehat{G}$. This takes time proportional to the sum of the degrees of all subvertices, which is again $O(n+k)$. The procedure maintains the loop invariant that the processed subvertices have valid edge rings, and hence at the end all the edge rings are valid and form a valid DCEL structure. This approach is theoretically optimal, but the DCEL structure of the overlay requires a large amount of memory because the overlay is usually much finer than the input meshes. Therefore we seek an alternative approach that does not require storing the DCEL structure.

\subsubsection{Enumerating subfacets}

The second approach is to enumerate the subfacets contained in a given blue or green facet. One can conveniently embed this procedure in a data transfer algorithm, 
or use it to enumerate all the subfacets by looping through all the blue or green facets.

The basic idea of the procedure is to use divide-and-conquer as illustrated in Figure 8 . We first create a list of the subvertices in the edges of the blue facet in counterclockwise order. Given a list, we take two adjacent subvertices in the list, walk along the subedges, and make a left turn whenever a subvertex is reached, until all subvertices in the list have been visited or the visited subvertices cut the list into two parts. In the former case, the list of subvertices determines a subfacet. In the latter case, we split the original list into two and call the procedure recursively on each of them. The time complexity for enumerating the subfacets in a blue or a green facet is $O(m \log m)$, where $m$ is the number of subvertices contained in this facet. In the worst case, $m$ can be $O(n)$, but for well-shaped meshes, which are of more practical interest, $m$ is bounded by a small constant. Therefore in practice enumerating all subfacets typically takes $O(n+k)$ time.

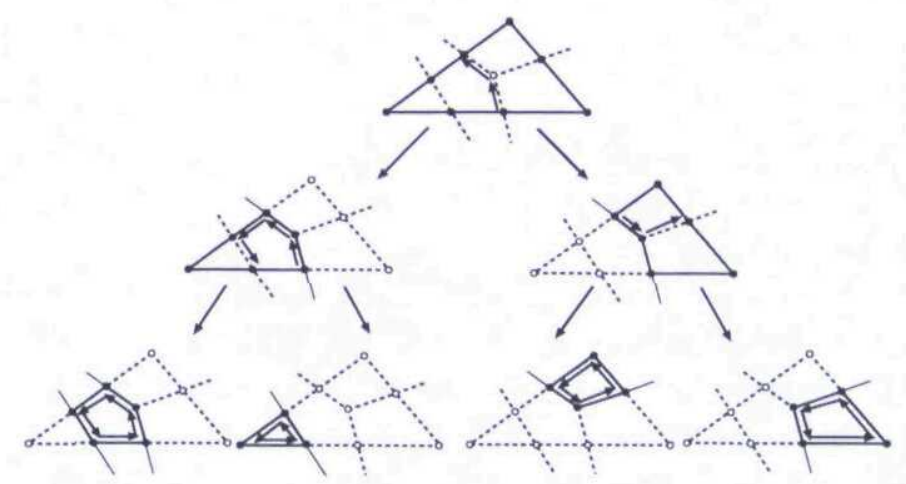

Fig. 8. Enumerating subfacets using divide-and-conquer. Dark subedges indicate current list of subvertices being processed, and arrows indicate traversal of subedges.

\section{Robust Primitives with Inexact Computations}

In the description of our algorithm, we have previously assumed that all primitives can be solved exactly, and the computation of edge intersection is reliable and gives exactly one valid solution. Unfortunately, these assumptions are unrealistic for practical meshes. Exact solution is impossible in general, because the primitives of our algorithm involve nonlinear equations that can be solved only approximately by iteration. Edge intersection may not always be reliable, because a blue and a green edge can be nearly parallel, causing the primitive to be very sensitive to perturbation. The inevitable presence of numerical errors introduces twofold difficulties to robustness: First, it can introduce inconsistencies into the solution and cause an algorithm to fail or produce invalid results. Second, it invalidates existing techniques 
for handling degeneracies (such as symbolic perturbation), because these techniques require exact computations. ${ }^{13,16,18}$ We first develop robust implementations of the primitives by monitoring errors and applying a perturbation scheme.

\subsection{Point projection}

Point projection involves solving a nonlinear equation using an iterative solver, Newton's method, whose stopping criterion is typically based on monitoring the residual of the equation. Under the assumptions of well-shapedness and near-orthogonality, the condition number of the equation is bounded. The error in the solution is bounded by the product of the condition number and the residual. ${ }^{29}$ For this particular situation, by specifying an a priori upper bound on the condition number, we can obtain an upper bound $\varepsilon$ on the error. If a point projects to a vertex or an edge within the tolerance $\varepsilon$, we then perturb the projection onto the vertex or edge, respectively. This perturbation technique is sometimes called tolerancing. For large enough $\varepsilon$, tolerancing guarantees that every blue/green point must project onto the interior or the boundary of at least one green/blue facet, and must not project onto the interior of more than one facet.

\subsection{Edge intersection}

Edge intersection is more subtle than point projection, because even if the meshes are well-shaped and smooth, a pair of blue and green edges can be nearly parallel to each other, which can lead to arbitrarily large condition numbers. It hence seems impossible to bound the errors in edge intersection. Furthermore, two nearly parallel edges can also cause the quadratic equation for this primitive to have zero or two solutions, but our algorithm requires it to return no more than one solution.

Fortunately, for well-shaped meshes, if $b$ and $g$ are nearly parallel, then there is a green edge incident on $g$ whose angle with $b$ is large, so that their edge intersection is well-conditioned, and vice versa. A robust implementation of our algorithm must avoid making any decision based on an unreliable intersection of $b$ and $g$, but should resort to the intersections of $b$ and incident edges of $g$. Therefore, we estimate the condition number of the primitive, and let the primitive report no intersection if the condition number is large or if there is more than one solution to the quadratic equation. A good estimate of the condition number for $\alpha$ is the reciprocal of the sine of the angle between $b$ and $l$, and for $\beta$ is that between $g$ and $\boldsymbol{n}$, where $\boldsymbol{l}$ and $n$ are defined in Section 3.2. For well-conditioned edge intersection, we then apply tolerancing to perturb an intersection onto a vertex if $\alpha$ (or $\beta$ ) is close to 0 or 1 .

\section{Resolution of Inconsistencies}

Unfortunately, the robustness of an algorithm does not follow automatically from robust implementations of primitives, because solutions of different primitives may contradict each other. Such inconsistencies must be identified and resolved properly to produce a valid overlay. 


\subsection{Location of subvertex}

Recall that our algorithm locates a subvertex on a blue edge $b$ by computing the intersection of $b$ with a set of candidate green edges that form a linear or cyclic sequence, where $b$ intersects them at either a single point or no point. In the presence of errors, the numerical solutions may report $b$ intersecting the green edges at zero or more than one intersection, even when there is one true intersection.

Figure 9 illustrates three different types of inconsistencies and their remedies. In the first two cases, numerical computations return two intersections between $b$ and the green edges, with the difference that in Case (a) one of the intersections is close to (or at) the origin of $b(\operatorname{org} b)$, whereas in Case (b) both intersections are far away from org $b$. Case (a) occurs when $\operatorname{org} b$ is too close to one of the green edges. We perturb org $b$ onto its nearby green edge (by reassigning the green parent of $\operatorname{org} b$ using the intersection principle in the next subsection), and then recompute the intersection of $b$ with a new set of candidate green edges. Case (b) occurs when $b$ is too close to the common vertex of the green edges that intersect $b$. (These green edges may not share a common vertex, but this would indicate that the green mesh is not well shaped or the projection is far from orthogonal.) We resolve this inconsistency by perturbing the intersection to the green vertex.

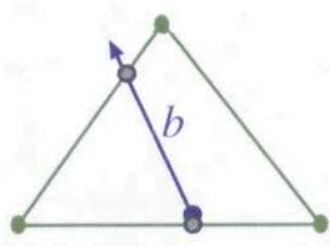

(a)

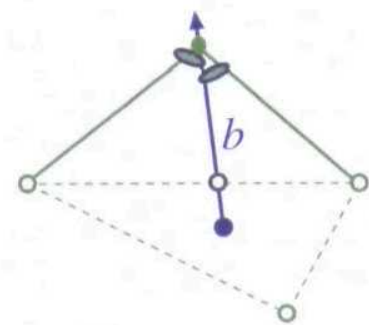

(b)

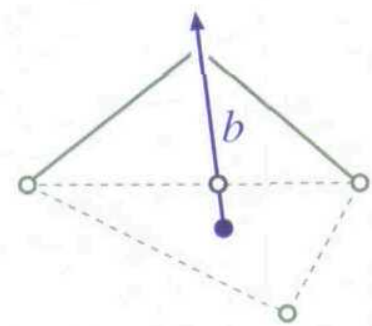

(c)

Fig. 9. Robust implementation of series of intersections. (a) Origin of blue (darker) edge is perturbed onto closest green (lighter) edge. (b) Blue edge intersects two green edges and is perturbed to green vertex. (c) Blue edge falls in artificial gap at green vertex and is also perturbed to vertex.

In Case (c), numerical errors cause $b$ to fall into an artificial gap at a green vertex between green edges so that no edge intersection is reported. As for Case (b), this case happens only when $b$ is too close to the green vertex, but Case (c) is more difficult because having no intersection can be a valid solution, in particular when dst $b$ is the next subvertex to locate. Case (c) differs from valid no-intersection cases in that $\alpha$ is between 0 and 1 for some edge intersections rather than being always greater than 1 . In Case (c), $\beta$ is less than zero for the green edges to its left and greater than 1 for the green edges to its right. We perturb the intersection to the green vertex if Case (c) is identified. 


\subsection{Inconsistencies among subvertices}

The parents of different subvertices can also be inconsistent with each other. For example, two subvertices in the interior of two blue edges may claim to coincide with the same green vertex. The perturbation schemes can even increase the likelihood of such inconsistencies. To address such inconsistencies, we first characterize the consistency requirements of the overlay and then design remedies for any inconsistencies so that they can be detected and resolved.

Let $b$ be a blue edge parameterized by $\boldsymbol{b}(\alpha)=\boldsymbol{b}_{0}+\alpha\left(\boldsymbol{b}_{1}-\boldsymbol{b}_{0}\right)$. Let $r_{0}=$ org $b, r_{1}, \ldots, r_{m}, r_{m+1}=$ dst $b$ be the subvertices in $b$.

Consistency Rule 1: The Host Candidate Lemma.

Consistency Rule 2: $\boldsymbol{b}^{-1}\left(r_{i}\right)<\boldsymbol{b}^{-1}\left(r_{i+1}\right)$ for $0 \leq i<m$.

Consistency Rule 3: Each blue vertex hosts one subvertex.

These three rules also apply symmetrically to the subvertices in green cells. Rule 1 characterizes the topological aspect of the consistency requirements. Rule 2 requires the numerical solutions to be consistent with the topological solutions. Rule 3 comes from the homeomorphism between $B$ and $G$. Note that Rule 1 has a symmetric property in the sense that the condition is satisfied for the subvertices in a directed edge $b_{0} b_{1}$ if and only if it is satisfied for the subvertices in its twin directed edge $\boldsymbol{b}_{1} \boldsymbol{b}_{0}$, as stated in the Implicit Candidate Lemma.

In the algorithm, we continually monitor whether these rules are satisfied after locating a new subvertex $v$. For well-shaped meshes with a nearly orthogonal projection, we observe that a violation of the consistency rules caused by numerical errors typically involves two extremely close neighbor subvertices contained in an input edge, say $r_{i}$ and $r_{i+1}$ in a blue edge $b$, such that either $\boldsymbol{b}^{-1}\left(r_{i}\right) \geq \boldsymbol{b}^{-1}\left(r_{i+1}\right)$ or $g_{i+1}$ falls into $\overline{g_{i}}$ with $i \neq 0$. We can resolve a violation of Rule 2 simply by perturbing the parameterizations of $r_{i}$ and $r_{i+1}$ on $b$ to recover $\boldsymbol{b}^{-1}\left(r_{i}\right)<\boldsymbol{b}^{-1}\left(r_{i+1}\right)$. Generally, we resolve an inconsistency by merging $r_{i}$ and $r_{i+1}$ into a subvertex $r_{i}^{\prime}$ whose blue/green parent is the intersection of the blue/green parents of $r_{i}$ and $r_{i+1}$, and reparameterizing $r_{i}^{\prime}$ in its parents. After merging, we then check $r_{i}^{\prime}$ with its known neighbor subvertices against the rules and resolve any further inconsistencies by merging. We refer to this remedy as the intersection principle. This principle effectively clusters a set of subvertices into a new subvertex whose blue/green parent is the intersection of the blue/green parents of the original subvertices. Figure 10 shows three examples in increasing order of complexity. In these examples, if a violation of the consistency rules is detected for $u_{1} u_{2}$, the intersection principle must be applied repeatedly to re-enforce consistency. The aggregated effect is that the subvertices labeled with $b, g$, and the $u_{i}$ are clustered into a single subvertex, and every subcell incident on these subvertices shrinks into another subcell. This principle turns out to be very effective for developing a robust and manageable implementation.

The intersection principle is valid for the following reasons. Numerically, merging 


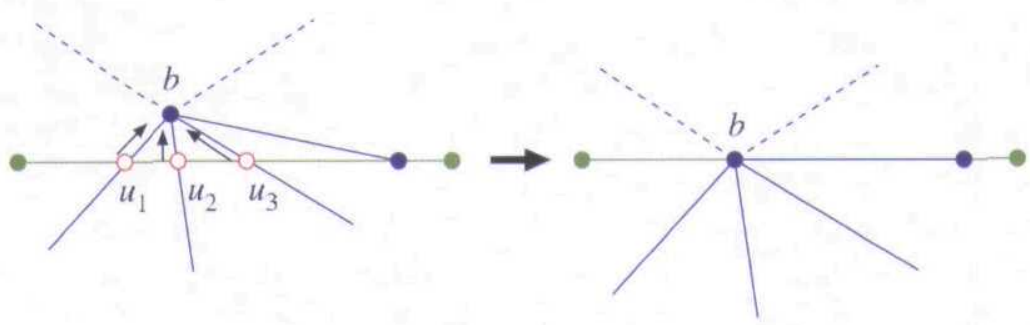

(a)
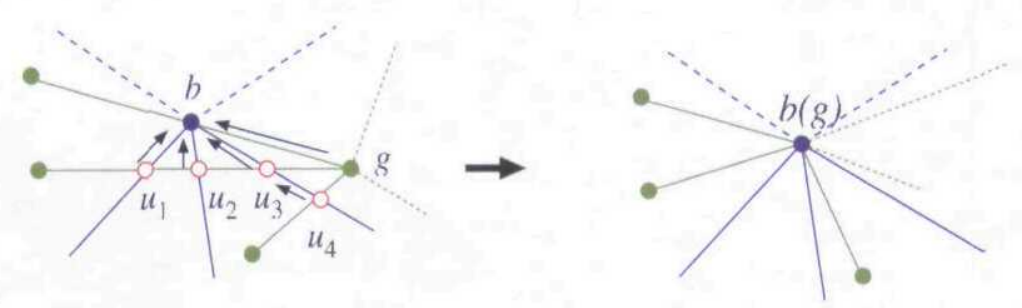

(b)
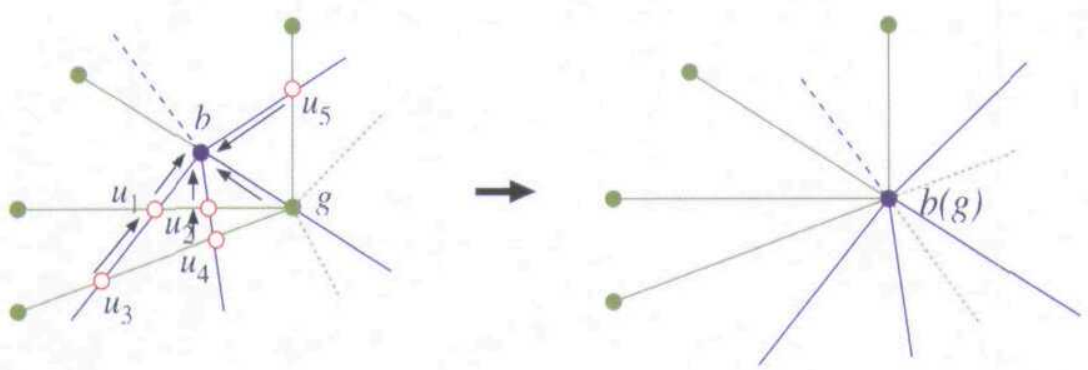

(c)

Fig. 10. Examples of clustering effect invoked by intersection principle.

two close subvertices eliminates some fine details in the overlay that the given precision cannot resolve. Topologically, because $g_{i} \cap g_{i+1} \leq g_{i} \in \mathrm{Cs} g_{i-1}$, the merging ensures that the green parent of $r_{i}^{\prime}$ is always in Cs $g_{i-1}$. Provided $g_{i} \cap g_{i+1} \neq \emptyset$, one can repeat the merging until the rules are re-enforced. This remedy fails only when the intersection of the blue or green parents of the violating subvertices becomes empty, which typically indicates that either the meshes are not well shaped or the 
projection is too far from orthogonal. The intersection principle delivers a consistent solution if the remedy succeeds. Intuitively, each clustering action invoked by the intersection principle can be replaced by a sequence of topology-preserving edge contractions on the overlay, which leads to a coarsened common refinement of $B$ and $G$. Although intuitively clear, the proof for topological preservation is rather involved, and we defer it to Part II.

\subsection{Implementation}

We now incorporate detection and resolution of inconsistencies into our algorithm. We define a procedure for "purifying" a subvertex by checking against the three consistency rules and resolving inconsistencies using the intersection principle. Whenever the algorithm locates a subvertex (Step 1 of Phase 1), it passes the subvertex to this procedure and uses the purified result to continue the computation. When sorting subvertices in green edges (Step 2 of Phase 1), we check whether each subvertex has only one predecessor and one successor. A violation of Rule 2 is detected if there is more than one, and we apply the intersection principle to resolve it. After all the subvertices have been sorted, we check and enforce Rule 1 for all green edges. When identifying the blue parents of green vertices (Step 3 of Phase 1), we also attain consistency by applying the intersection principle if a green vertex projects onto an edge or a vertex. Phase 2 of the overlay algorithm (i.e., the construction of the subfacets) is purely combinatorial and requires no special treatment for consistency. In our experience, it is generally sufficiently robust to implement inconsistency checking and resolution only for Step 1 of Phase 1, and to consider only adjacent subvertices when checking Rule 1 and invoke checking only when the parameterizations of two adjacent subvertices are very close to each other.

Due to error checking and consistency enforcement, our implementation in general requires $O(d(n+k))$ time instead of the optimal $O(n+k)$, where $d$ is the maximum degree of the subvertices, and $n$ and $k$ are input and output sizes, respectively. For well-shaped meshes, $d$ is typically a small constant, and the practical performance of this implementation is still linear in its input plus output size.

We have implemented the above algorithm in a software package Rocface, the interface component of the integrated rocket simulation code (Rocstar) at the Center for Simulation of Advanced Rockets at the University of Illinois. The implementation is in $\mathrm{C}++$ with double-precision arithmetic, using the half-edge data structure of the CGAL library. ${ }^{30}$ To improve reliability for complex geometries with ridges and corners, Rocface includes extensions to the algorithm for detecting and matching such features of input meshes. We will present experimental results for the extended algorithm in Part II.

\section{Discussion}

This paper addressed the mesh overlay problem in a general and complex setting. We recognized that two meshes modeling the same surface can have not only dif- 
ferent combinatorial structures, but also different geometric realizations. We gave a precise definition for an overlay of surface meshes with different geometric realizations, and presented a new definition and solution techniques for the intersection of edges in different mesh surfaces. When designing the algorithm, we considered surface meshes of most practical interest, namely well-shaped triangular and quadrilateral meshes. In this complex setting, we presented an algorithm that requires linear time and space. These realistic situations lead to nonlinear systems for the primitives, and therefore inexact computations and explicit treatments of degeneracies are inevitable. We described how to implement our algorithm for overlaying surface meshes robustly even with inexact computations, through a combination of techniques, including error analysis, perturbation, and detection and resolution of inconsistencies. In contrast to traditional perturbation schemes, we perturb near degeneracies to true degeneracies and explicitly address degeneracies in the algorithm. Our approach is fairly complicated and may not be applicable to other geometric problems for which the consistency requirements cannot be easily identified. However, we believe our approach can benefit at least a few other applications.

The overlay mesh has many potential applications in scientific computing and computer graphics. One major application is to transfer data between nonmatching meshes. When applying the overlay to data transfer, we further triangulate its subfacets to obtain a triangular common refinement of the meshes. The availability of this triangular common refinement enables a class of weighted residual data transfer methods that are strictly conservative and more accurate than traditional methods. ${ }^{1}$ As future work, we plan to extend the techniques used in this paper to overlay volume meshes so that data can be transferred accurately after adaptive mesh refinement, coarsening, or remeshing. Note that surface overlay is a necessary step for performing volume mesh overlay because in general the boundaries of volume meshes are nonmatching surface meshes. The algorithm can also be extended to handle cases where two mesh surfaces partially overlap, which also arise frequently in practice

This paper presented only a sequential algorithm for computing the subdivision. The algorithm has rich potential for parallelism because all operations are local and in principle can be done in parallel with little communication. The parallelization is by no means trivial, however, for the following reasons. First, the input meshes can be partitioned differently from each other, and hence one must redistribute at least one of the input meshes based on the partitioning of the other mesh. Second, to resolve inconsistencies caused by numerical errors near partition boundaries, some coordination is needed among processors. Third, multiple numbering systems for vertices and facets are involved, which introduces additional complexities. Fortunately, the sequential algorithm presented in this paper is very efficient, and it takes a very small fraction of the mesh generation time. Furthermore, a common refinement needs to be constructed only after meshing or remeshing, but the data transfer that uses it is performed repeatedly for every time step of a physical simulation. Therefore, construction of the common refinement is rarely a bottleneck, and the 
sequential algorithm almost always suffices in practice. We leave the parallelization of mesh overlay as future work.

\section{Acknowledgments}

This research was supported by the Center for Simulation of Advanced Rockets funded by the U.S. Department of Energy under Subcontract B341494. The authors thank Professor Herbert Edelsbrunner for helpful discussions on the topic of overlay. We thank anonymous referees for their helpful comments in improving the presentation of the paper.

\section{References}

1. X. Jiao and M. T. Heath, Common-refinement based data transfer between nonmatching meshes in multiphysics simulations, Int. J. Numer. Meth. Engrg. (2004), to appear.

2. J. R. Cebral and R. Löhner, Conservative load projection and tracking for fluidstructure problems, AIAA J. 35 (1997) 687-692.

3. J. K. Dukowicz, Conservative rezoning (remapping) for general quadrilateral meshes, J. Comput. Phys. 54 (1984) 411-424.

4. J. D. Ramshaw, Conservative rezoning algorithm for generalized two-dimensional meshes, J. Comput. Phys. 59 (1985) 193-199.

5. T. Kanai, H. Suzuki and F. Kimura, Metamorphosis of arbitrary triangular meshes, IEEE Comput. Graph. \& Appl. 20 (2000) 62-75.

6. M. Alexa, Merging polyhedral shapes with scattered features, Visual Comput. 16 (2000) 26-37.

7. J. R. Kent, W. E. Carlson and R. E. Parent, Shape transformation for polyhedral objects, Comput. Graph. 26 (1992) 47-54.

8. M. de Berg, M. van Kreveld, M. Overmars and O. Schwarzkopf, Computational Geometry: Algorithms and Applications, 2nd edition (Springer, Berlin, 2000).

9. I. J. Balaban, An optimal algorithm for finding segments intersections, in Proc. 11th Ann. ACM Sympos. Comput. Geom., Vancouvar, Canada (June 1995) pp. 211-219.

10. B. Chazelle and $H$. Edelsbrunner, An optimal algorithm for intersecting line segments in the plane, J. ACM 39 (1992) 1-54.

11. U. Finke and K. H. Hinrichs, Overlaying simply connected planar subdivisions in linear time, in Proc. 11th Ann. ACM Sympos. Comput. Geom., Vancouvar, Canada (June 1995) pp. 119-126.

12. L. J. Guibas and R. Seidel, Computing convolutions by reciprocal search, Disc. \& Comput. Geom. 2 (1987) 175-193.

13. H. Edelsbrunner and E. P. Mücke, Simulation of simplicity: a technique to cope with degenerate cases in geometric algorithms, ACM Trans. Graph. 9 (1990) 66-104.

14. S. Fortune, Robustness issues in geometric algorithms, in Applied Computational Geometry, eds. M. C. Lin and D. Manocha (Springer, Berlin, 1996) pp. 9-14.

15. L. Guibas, D. Salesin and J. Stolfi, Epsilon geometry: building robust algorithms from imprecise computations, in Proc. 5th Ann. ACM Sympos. Comput. Geom., Saarbruchen, Germany (June 1989) pp. 208-217.

16. C. M. Hoffmann, J. E. Hopcroft and M. S. Karasick, Towards implementing robust geometric computations, in Proc. 4th Ann. ACM Sympos. Comput. Geom., UrbanaChampaign, IL (June 1988) pp. 106-117.

17. V. J. Milenkovic, Verifiable implementations of geometric algorithms using finite precision arithmetic, Artifi. Intell. 1 (1988) 377-401. 
18. C. K. Yap, Symbolic treatment of geometric degeneration, J. Symb. Comput. 10 (1990) 349-370.

19. M. T. Goodrich, L. J. Guibas, J. Hershberger and P. J. Tanenbaum, Snap rounding line segments efficiently in two and three dimensions, in Proc. 13th Ann. ACM Sympos. Comput. Geom., Nice, France (June 1997) pp. 284-293.

20. D. Halperin and E. Packer, Iterated snap rounding, Comput. Geom. Theory Appl. 23 (2002) 209-225.

21. E. D. Bloch, A First Course in Geometric Topology and Differential Geometry (Birkhäuser, Boston, 1997).

22. H. Edelsbrunner, Geometry and Topology for Mesh Generation (Cambridge University Press, Cambridge, 2001).

23. L. Kobbelt, T. Bareuther and H.-P. Seidel, Multiresolution shape deformations for meshes with dynamic vertex connectivity, in Proc. EUROGRAPHICS 2000, Interlaken, Switzerland (Aug. 2000) pp. 249-260.

24. D. P. Huttenlocher and K. Kedem, Computing the minimum Hausdorff distance for point sets under translation, in Proc. 6th Ann. ACM Sympos. Comput. Geom., Berkeley, CA (June 1990) pp. 340-349.

25. L. Kettner, Designing a data structure for polyhedral surfaces, in Proc. 14th Ann. ACM Sympos. Comput. Geom., Minneapolis, MN (June 1998) pp. 146-154.

26. L. J. Guibas and J. Stolfi, Primitives for the manipulation of general subdivisions and the computation of Voronoi diagrams, ACM Trans. Graph. 4 (1985) 74-123.

27. B. G. Baumgart, A polyhedron representation for computer vision, in Proc. Natl. Comput. Conf. 44 (AFIPS Press, NJ, 1975) pp. 589-596.

28. X. Jiao, H. Edelsbrunner and M. T. Heath, Mesh association: formulation and algorithms, in 8th Int. Meshing Roundtable, South Lake Taho, CA, (1999) pp. 75-82.

29. M. T. Heath, Scientific Computing: An Introductory Survey, 2nd edition (McGrawHill, New York, 2002).

30. S. Schirra, R. Veltkamp and M. Yvinec, CGAL basic library reference manual (http://www.cgal.org, 2000).

\section{Appendix A. Proof of Pairwise Intersections Lemma}

Proof. Presuming the lemma is true, we refer to the closures of the 0-, 1-, and, 2-dimensional intersections as subvertices, subedges, and subfacets, respectively. It is easy to see that these subcells form a pure complex, which requires that (1) each subfacet is a polygon with a finite number of edges, (2) each subedge or subvertex belongs to at least one subfacet, and (3) any two subfacets either are disjoint, intersect at a single subvertex, or intersect along a single subedge. The first two conditions are trivial. For the third condition, consider two subfacets $b_{1} \cap_{f} g_{1}$ and $b_{2} \cap_{f} g_{2}$, where $b_{1}$ and $b_{2}$ are their blue parents, $g_{1}$ and $g_{2}$ are their green parents, and either $b_{1} \neq b_{2}$ or $g_{1} \neq g_{2}$, or both. Their intersection is contained in $\left(b_{1} \cap_{f} g_{1}\right) \cap\left(b_{2} \cap_{f} g_{2}\right)=\left(b_{1} \cap b_{2}\right) \cap_{f}\left(g_{1} \cap g_{2}\right)$, which is either the empty set, a subvertex, or a subedge.

Let $R$ denote the complex formed by the subcells. Because $|R|$ is homeomorphic to both $|B|$ and $|G|$, and each subcell is contained in a cell in $B$ and a cell in $G$, by definition $R$ is a common refinement of $B$ and $G$. To show it is a minimal common refinement, suppose two distinct subcells $\sigma$ and $\tau$ share the same blue and the same 
green parents that intersect in a subcell $\varphi$ in $R$. Then, either $\sigma$ or $\tau$ must be in the boundary of $\varphi$ and in turn must be contained in the boundary of its blue or green parent, which contradicts the definition of the parent of a subcell.

\section{Appendix B. Proof of Host Candidate Lemma}

Proof. From the continuity of the homeomorphism, it is obvious that $g_{i+1} \in$ $\overline{\mathrm{St} g}$. Therefore, we just need to show that

$$
\begin{gathered}
g_{i+1} \notin \operatorname{St} g_{j} \text { for } 0 \leq j \leq i<m, \\
g_{i+1} \notin \overline{g_{i}} \text { for } 0 \leq j \leq i<m, \\
g_{i+1} \notin \overline{\operatorname{St} g_{j}} \text { for } j<i \leq m .
\end{gathered}
$$

By the definition of conformality, we know that $g_{i+1} \neq g_{j}$ for $0 \leq j \leq i<m$. To prove $g_{i+1} \notin$ St $g_{j}$ (B.1), we consider the cases where $g_{j}$ is a facet, an edge, or a vertex separately. If $g_{j}$ is a facet, then its star is $g_{j}$ itself, which cannot be $g_{i+1}$. If $g_{j}$ is an edge, its star is $g_{j}$ itself plus its incident facets, but $g_{i+1}$ must not be a facet, and hence again $g_{i+1} \notin \mathrm{St} g_{j}$. If $g_{j}$ is a vertex, its star contains $g_{j}$, its incident edges, and incident facets. The facets are ruled out as above. The incident edges cannot be $g_{i+1}$ because if two edges intersect along an interval, then both of its endpoints must be blue or green vertices.

Similarly, for (B.2) note that $g_{i}$ for $0<i \leq m$ is either a vertex or an edge. If $g_{i}$ is a vertex, then $g_{i+1} \in \overline{g_{i}}$ implies $g_{i+1}=g_{i}$, which contradicts the homeomorphism. If $g_{i}$ is an edge, then $g_{i+1} \in \overline{g_{i}}$ implies that the blue edge intersects $g_{i}$ along an interval whose endpoints must be blue or green vertices. This contradicts the fact that $r_{i}$ is neither a blue nor a green vertex.

Finally, we need to show (B.3). Notice that the blue edge $b$ intersects the facets in $\overline{\mathrm{St} g}$ either at a point or along an interval. For a conforming homeomorphism, the intersection of a blue and a green cell must be connected, which implies that $g_{i+1} \in \overline{\mathrm{St} g_{j}}$ only if $j=i$ or $j=i+2$.

\section{Appendix C. Proof of Implicit Candidate Lemma}

Proof. We use the following facts in our proof:

$$
\begin{aligned}
\sigma \in \overline{\mathrm{St}} \tau & \Leftrightarrow \tau \in \overline{\mathrm{St} \sigma}, \\
\sigma \in \bar{\tau} & \Leftrightarrow \tau \in \mathrm{St} \sigma, \\
\sigma \in \mathrm{Cs} \tau & \Rightarrow \tau \in \overline{\operatorname{St} \sigma}-\bar{\sigma} .
\end{aligned}
$$

In particular, observe that

$$
\begin{aligned}
g_{i+1} \in \overline{\mathrm{St} g_{i}} & \Rightarrow g_{i} \in \overline{\mathrm{St} g_{i+1}}, \\
g_{i+1} \notin \overline{g_{i}} & \Rightarrow g_{i} \notin \mathrm{St} g_{i+1},
\end{aligned}
$$




$$
\begin{aligned}
& g_{i+1} \notin \text { St } g \Rightarrow g_{i} \notin \overline{g_{i+1}}, \\
& g_{j>i+1} \notin \overline{\text { St } g_{i}} \Rightarrow g_{i} \notin \overline{\text { St } g_{j>i+1}} .
\end{aligned}
$$

For $i=0 \neq m$, from (C.1), (C.3), and (C.4), we have

$$
g_{i} \in \overline{\operatorname{St} g_{i+1}}-\overline{g_{i+1}}-\bigcup_{j>i+1} \overline{\operatorname{St} g_{j}} .
$$

For $i=m$, from (C.1), (C.2), we have

$$
g_{i} \in \overline{\text { St } g_{i+1}}-\text { St } g_{i+1}=\operatorname{Cs} g_{i+1} \text {. }
$$

For $0<i<m$, from (C.1), (C.2), (C.3), and (C.4), we have,

$$
g_{i} \in \operatorname{Cs} g_{i+1}-\overline{g_{i+1}}-\bigcup_{j>i+1} \overline{\operatorname{St} g_{j}} .
$$


Copyright of International Journal of Computational Geometry \& Applications is the property of World Scientific Publishing Company and its content may not be copied or emailed to multiple sites or posted to a listserv without the copyright holder's express written permission. However, users may print, download, or email articles for individual use. 